\title{
Les figures du musicien au Moyen Âge. Figures, discours et images
}

Centre d'études médiévales d'Auxerre, 24-25 mai 2007

\section{Martine Clouzot}

\section{(2) OpenEdition}

\section{Journals}

Édition électronique

URL : https://journals.openedition.org/cem/1552

DOI : $10.4000 /$ cem. 1552

ISSN : 1954-3093

Éditeur

Centre d'études médiévales Saint-Germain d'Auxerre

Édition imprimée

Date de publication : 15 août 2007

ISSN : 1623-5770

\section{Référence électronique}

Martine Clouzot, «Les figures du musicien au Moyen Âge. Figures, discours et images », Bulletin du centre d'études médiévales d'Auxerre | BUCEMA [En ligne], 11 | 2007, mis en ligne le 29 août 2007, consulté le 22 septembre 2022. URL : http://journals.openedition.org/cem/1552 ; DOI : https://doi.org/ 10.4000/cem. 1552

Ce document a été généré automatiquement le 22 septembre 2022.

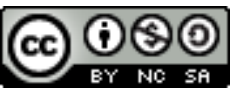

Creative Commons - Attribution - Pas d'Utilisation Commerciale - Partage dans les Mêmes Conditions 4.0 International - CC BY-NC-SA 4.0

https://creativecommons.org/licenses/by-nc-sa/4.0/ 


\section{Les figures du musicien au Moyen Âge. Figures, discours et images}

Centre d'études médiévales d'Auxerre, 24-25 mai 2007

Martine Clouzot

1 L'idée d'un atelier sur «Les figures du musicien au Moyen Âge » est née à la Casa de Velasquez à Madrid en juin 2005, lors du colloque organisé par Eliana Magnani sur « Le Moyen Âge vu d'ailleurs ». Je la remercie d'avoir favorisé ainsi des collaborations scientifiques fructueuses et prometteuses, qui se concrétisent grâce au Centre d'études médiévales d'Auxerre et à l'UMR 5594 ARTeHis de l'Université de Bourgogne.

Les discussions de cet atelier se sont articulées autour de la question à laquelle les musicologues sont rompus et habitués à répondre : qu'est-ce qu'un musicien? Cette question a d'emblée été pensée au pluriel, car le terme recouvre une typologie complexe de termes et de formes. Elle s'est posée en trois temps : d'abord, sur le mot, le concept, la figure; puis sur les usages qui en sont faits selon les contextes; enfin dialectiquement, en confrontant les vocables et leurs usages insérés dans leur contexte historique. Dès lors, la mention du « musicien » dans un document, qu'il soit d'archives, narratif, figuratif, qu'il soit l'objet lui-même, relève-t-elle d'un concept, d'une idée, a-telle un usage? Quelles significations et quelles finalités ce mot, cette figure, ce personnage revêtent-ils dans le document, dans la société, dans la pensée des auteurs qui les évoquent? Le terme n'apparaissant pas, semble-t-il, dans les documents médiévaux en français, nous sommes partis du latin : le musicus.

Musicus, cantor et poète

Chez saint Augustin et chez Boèce, le musicus est l'homme libre qui maîtrise l'art libéral de la musica d'où il tire son nom. À partir de l'orphisme pythagoricien, puis de Platon et Aristote, ces deux autorités n'ont jamais défini la musica comme une pratique de l'art musical, mais comme un ordre transcendant que l'on approche par la spéculation théorique, par la theoria au sens de "contemplation " ${ }^{1}$. Elle est conçue comme activité spirituelle qui repose sur la contemplation des principes mêmes de cet art. Dans l'ordre antique des disciplines du savoir, la musica est la science du Nombre, du numerus et fait partie du quadrivium, des sciences mathématiques qui mesurent et relient le monde 
terrestre et céleste. C'est pourquoi Boèce le platonicien a enseigné au Moyen Âge à penser non pas sur la musique, mais selon la musique, selon ses principes et ses signes : les trois musiques boéciennes exposent cette conception universelle: la musica instrumentalis qui est produite par l'homme et la technique instrumentale et la voix, la musica humana qui se révèle à l'homme lorsqu'il descend en lui-même et découvre dans son microcosme les lois qui gouvernent le macrocosme, enfin la musica mundana dont l'harmonie gouverne l'univers ${ }^{2}$ - c'est le Timée de Platon.

Parallèlement à cette classification tripartite, Boèce a divisé l'art musical en trois types de "musiciens ", dans le chapitre intitulé "Quid sit musicus» du De Musica. Le premier genre exclut les joueurs d'instruments de la spéculation musicale - c'est en quelque sorte le héraut d'armes, instrumentiste, originaire du monde des ménestrels, que Maria NARBONA a étudié à la cour de Charles III roi de Navarre au XIV ${ }^{\mathrm{e}}$ siècle. Le deuxième genre de musicus boécien regroupe les poètes qui composent par instinct naturel plutôt que par spéculation; leurs poésies restent alors en dehors de la musique - nous trouvons là le jongleur de Silvère MENÉGALDO et les poètes du Tournois de Chauvency de Jean-Marie FRITZ. Enfin le $3^{e}$ genre est celui du musicien authentique qui pense selon la musique, qui est capable de juger des modes, des rythmes, des chansons, des poèmes, incarné par les "figures de compositeurs » présentées par Eduardo AUBERT, Étienne ANHEIM, David Fiala, Gregor METZIG. Toutefois, il sera nécessaire de nuancer la part de la pensée boécienne dans la culture musicale au tournant des XIII et XIV ${ }^{\mathrm{e}}$ siècles.

5 L'étude d'Eduardo AUBERT sur «Figuration de la musique et musicalité de la figure : les miniatures et les neumes dans le tonaire d'Auch (BnF, ms. lat. 1118)» a en effet démontré de façon très convaincante le système dynamique et cohérent, visuel et sonore, qui structure d'une part l'écriture, l'iconographie, le chant, d'autre part les rapports de lecture dynamique et de geste musical entre le manuscrit et son lecteur. On peut penser, comme l'a proposé Daniel Russo, que le manuscrit a été conçu comme un objet-medium, dont l'iconographie «iconise » les mouvements du chant selon Eduardo Aubert. En avançant dans le temps, Étienne Anheim a mis en évidence le " compositeur-auteur» dont la figure émerge au tournant des années 1275 à Paris autour du fameux traité de l'Anonyme $I V$, qui convoque les figures d'autorité du "compositeur" que sont Léonin, Pérotin; annonçant ainsi la "figure d'auteur" revendiquée, identifiée, exemplifiée et intériorisée, notamment par Guillaume de Machaut, Pétrarque. Étienne Anheim a montré la genèse du statut de compositeur, à partir du verbe componere (et non du mot compositor) dans l'espace canonial, puis curial $\mathrm{du} \mathrm{XIV}^{\mathrm{e}}$ siècle. Au XV ${ }^{\mathrm{e}}$ siècle, le « compositeur" citant son nom dans ses œuvres est interprété, à juste titre, par David Fiala, comme une "figure musicale de la nomination" qui relève d'une mise en scène de l'individu, d'autoportrait, d'autobiographie musicale, notamment chez Guillaume Du Fay, Gilles Binchois, Ockeghem, Johannes Tinctoris, Josquin Desprez.

D'autres usages de la parole musicienne transitent par le «musicien», certes souvent au détriment de l'art musical, mais au profit d'une autre harmonie, sociale et politique. Le «musicien » est aussi le poète qui, pour composer, joint l'expérience de la theoria musicale à la connaissance technique du langage et à une sagesse inspirée ${ }^{3}$. Le numerus est dans ce contexte l'équivalent latin du grec rhythmus; tous deux introduisent une « rationalité musicale » dans l'écriture et la parole poétiques, par le mouvement réglé des vers, des rimes, des sonorités. C'est le mariage des arts ${ }^{4}$, et en particulier celui de la musique du quadrivium avec la grammaire du trivium qui, depuis l'Antiquité, et surtout 
depuis Augustin et Boèce, unit le musicus au poète. Empreinte et science de la lettre, (gramma), la grammaire rend audible la parole mesurée par le rythme et le nombre, elle donne corps à l'écriture musicale par ses signes et ses figures. Le poète, ou plus exactement le «facteur», le "faiseur", «l'ouvrier» (aristotélicien), mais aussi "l'acteur", "l'aucteur", le "rimeur", le "versifieur", sont autant de termes pour nommer un autre «musicien» dont les activités sont orientées vers l'action "agere». Le " poète » désigne ses ancêtres de l'Antiquité, les devins de la fable, les prophètes le vates. Mais l'art du «faiseur » à partir du milieu du XIV e siècle s'appelle la poésie ou " poétrie ", qui raconte les histoires (fictives) les plus nobles, elles sont empruntées à la mythologie antique, à la "fiction » mythique dont le poète réactualise la mémoire. Le poète Jacques Bretel a composé son récit du Tournois de Chauvency (1285, Lorraine) comme une partition musicale, en jouant sur une large palette sonore de cris de hérauts, d'instruments de musique, de chants, de bruits de bataille, mais aussi de rythmes et de couleurs : le manuscrit est par le texte lyrique, les images et l'héraldique, un objet musical. Jean-Marie Fritz a fort bien montré comment Jacques Bretel s'est mis en scène tout au long du poème à la fois comme "narrateur ", «auteur " du livre, spectateur et «acteur» du tournoi. De même, le héraut d'armes analysé par Maria Narbona joue un rôle central dans l'économie politique et cérémonielle de la cour : maître de cérémonie, il est un " maître de la parole » qui annonce, par la voix et au son de la trompette, les familles de la noblesse dont il crie et décrit les armoiries, les situant ainsi selon un protocole hiérarchique à la cour et dans la société aristocratique. Des compositeurs de haut niveau ont contribué idéalement à l'harmonie politique. Heinrich Isaac au service du pape Léon X, puis « maître de musique » de l'empereur germanique Maximilien $\mathrm{I}^{\mathrm{er}}$, illustre le statut politique du musicus à travers le rôle diplomatique de la musique : Gregor Metzig a démontré que la composition des motets pour l'empereur est une action politique qui vaut comme moyen de communication entre Maximilien $\mathrm{I}^{\mathrm{er}}$ et Léon X.

7 En revanche, il est un autre type de «musicien » qui ne favorise pas l'harmonie sociale et universelle, alors qu'il use des mêmes « outils », la parole, l'instrument, la mémoire : c'est le jongleur dont Silvère Menégaldo a analysé les très rares occurrences dans la lyrique d'oïl en posant la question du statut du poète et de son œuvre; pour saisir le mot et le personnage il faut se référer à d'autres éléments thématiques, tels que l'amour, la nature, la sincérité, la musique, la rétribution, la folle vie, la joie de vivre. Le jongleur tire ses origines des auteurs antiques. Tour à tour histrion virtuose dans la tradition de saint Jérôme, de saint Augustin, il est le concurrent du troubadour et du trouvère à partir du XII ${ }^{e}$ siècle, concurrent dans l'action de "trover ", de tropare, c'està-dire de "tourner le langage en figure », en versus, de "renouveler la forme d'un texte ou d'une mélodie ", « d'inventer ", de " trouver » ${ }^{5}$. Ses paroles sont à l'image de son jeu d'acteur: une gesticulatio terme qui a été violemment pris à partie tout au long du Moyen Âge. Contrairement au vrai poète, le jongleur est un imitateur qui n'a pas la mémoire exacte des histoires et des gestes mythiques. Il raconte avec son corps, avec sa voix et sa musique, une histoire au sens romain d'istoria, c'est-à-dire de spectacle dont les risques d'excès et d'idolâtrie ont provoqué les condamnations des moralistes, depuis Tertullien, puis saint Augustin. Le jongleur jongle avec sa voix et son corps, il chante. Il trouve en bien des points son prolongement à la Renaissance italienne chez le cantimbanco décrit par Florence ALAZARD comme le chanteur de la place publique, musicien rangé parmi les oisifs et les truands, tout en remplissant les rôles de médiateur des cultures communes et partagées entre les élites et le peuple, de porte- 
voix des citadins et de figure politique. Dès lors, le jongleur, serait-il une figure, non du jongleur en tant que personnage social, mais une figure de rhétorique : une figure du mot joculator (jongler avec les mots, les sons, les rimes), une figure de sens (tropes), une figure de style, d'éloquence corporelle, une figure de construction (qui joue sur l'inversion - des mots et du corps), une figure de pensée (allégorique, ironique)? Comment et pourquoi sert-il à transmettre des discours sur l'âme, le corps, le salut, le plaisir, le savoir, la mémoire, l'ordre social ?

Figures et discours

De la figure étymologique à la figure rhétorique, nous pouvons questionner le «musicien» sur ses autres figures, celle qui transcrivent leur aspect visible, leur configuration visible. Les «musiciens » sculptés dans le bois des stalles de chœur des $\mathrm{XV}^{\mathrm{e}}$ et $\mathrm{XVI}^{\mathrm{e}}$ siècles, présentés par Frédéric BILLIET à partir de sa riche base de données, appartiennent-ils à des thèmes iconographiques de la musique, sont-ils la «représentation» d'un motif anecdotique, proverbial, historique (au sens de récit) guidée par des codes iconographiques et des attributs? Par leur fonctionnement même, par leur matérialité, leur mise en scène, l'agencement de leurs formes, les images des "musiciens » ont-elles une finalité, remplissent-elles une fonction ${ }^{6}$, ont-elles une efficacité symbolique, provoquent-elles des effets? Dépassent-elles leur aspect formel pour servir un discours autre? À partir du livre d'Heures de Jeanne d'Evreux (New York, Cloisters Museum, ms. 54.1.2., vers 1325) et d'un missel conservé à Montpellier (BM, ms. 261, vers 1350), enluminés par Jean Pucelle, Béatrice BEYs a évoqué les «musiciens » hybrides et grotesques des peintures marginales. À partir de cet appareil figuratif qui encadre les feuillets, Daniel Russo a soulevé le problème essentiel du statut de l'intrument en rapport à ces livres et à ce types de "musiciens" : figure-t-on la musique pour elle-même? Dans ce cas de figures de musiciens ne serait-il pas en effet question, d'après notre perception, de défigurer plus que de figurer, ou bien "figurer» signifie aussi « défigurer». D'où l'interprétation que donne Georges Didi-Huberman du verbe "figurer ${ }^{7}$ : figurer une chose, c'est la signifier par autre chose que son seul aspect visible et extérieur.

9 Les «figures" des musiciens sont certainement avant tout des modes d'accès à la compréhension d'autres formes de discours. Elles inviteraient alors à penser et à parler selon la musique, pour reprendre la formule de Boèce, et non sur les musiciens. Car les discours dépassent les «figures " tant qu'ils traitent non des musiciens en eux-mêmes, mais de la musique et de son fondement pythagoricien et platonicien: le nombre, comme principe et comme action: le mouvement rythmé. La transmission de ce principe rythmique fondamental s'est portée sur la figure des signes - l'écriture musicale, les mouvements de la main guidonienne -, sur la production des sons (la voix, les instruments) et sur leur écoute (leur réception, leur compréhension rationnelle, leur pouvoir sur les sens et les émotions). La spéculation musicale emprunte à partir du $\mathrm{XIV}^{\mathrm{e}}$ siècle d'autres voies pour manifester l'harmonie universelle, le nombre en mouvement harmonieux et bien réglé. Avec « les engrenages musiciens. L'horloge et la figuration de la musique de l'univers au Moyen Âge », Philippe CORDEZ a donné à voir des objets "merveilleux» alliant des images cinétiques à une musique audible de l'harmonie divine. Les horloges astronomiques (celles de Gdansk, Lund, Bourges) les automates d'horloges (le coq, le papotier et le gueulard de Strasbourg) et l'orgue, sont tout à la fois musica, rythme, mouvement mesuré, musici. Ces engrenages ne forment-ils pas un bel exemple de "figure» dépassée par sa propre fonction - mesurer les 
mouvements du temps, indiquer l'heure -, de figure transformée par ce qu'elle "préfigure »: sur terre, sous l'aspect visible d'une image mécanique, les automates «préfigureraient » l'harmonie du monde et de son «horloger»: la figure de Dieu musicien. Ils rappellent, par leur matérialité mathématique et sonore, que la musica est liée à l'astronomie dans le quadrivium et qu'elle est un rapport entre "tout». Expérience théorique et technique, elle relie la terre au ciel, en invitant l'homme à contempler la beauté de l'harmonie universelle.

Cette présentation et cette synthèse générales des définitions de la musica et du musicus, visent à réfléchir sur un objet commun d'étude - les figures du musicien -afin d'en dégager les discours que transmettaient ces figures au Moyen Âge et ce qu'elles nous ont transmis à nous historiens de l'art, musicologues, historiens de la littérature et historiens. En partant de la musique, de sa place dans la pensée et dans la société médiévales, les « figures des musiciens » nous invitent à réfléchir sur la nature, sur la mémoire et sur les objets.

11 Chacun de ces termes pouvant être pensé et relié selon la musique, ils constitueront les axes d'un prochain atelier en 2009.

\section{Rappel du programme :}

- Florence ALAZARD, Le cantimbanco, figure du musicien dans les villes de la Renaissance italienne.

- Etienne AnHeim, La figure du compositeur entre le XIII et le début du XVe siècle.

- Eduardo AUBERT, Figuration de la musique et musicalité de la figure : les miniatures et les neumes dans le tonaire d'Auch (Paris, BnF, ms. lat. 1118).

- Béatrice BEYs, Vers le Paradis ou l'Enfer : les musiciens des marges de Jean Pucelle et de ses épigones.

- Frédéric BILLIET, Les figures musiciennes dans les stalles de chœur.

- Martine CLouzot, Le jongleur, serviteur ou miroir du prince aux XIII et XIV siècles ?

- Philippe CORDEZ, Les engrenages musiciens. L'horloge et la figuration de la musique de l'univers au Moyen Âge.

- David FIALA, Le nom propre en musique : noms de compositeurs dans leurs œuvres de Du Fay à Josquin.

- Jean-Marie FRITZ, Figures du musicien dans le Tournoi de Chauvency de Jacques Bretel.

- Silvere MÉNÉGALDO, Les « Figures du jongleur dans la lyrique d'oïl »

- Gregor METZIG, Heinrich Isaac (1450-1517). Musique et Diplomatie au service de l'empereur Maximilien $\mathrm{I}^{\mathrm{er}}$.

- Maria NARBONA CÀrCELES, Le héraut d'armes dans les officines d'armes en Europe à la fin du XIV siècle.

\section{NOTES}

1. R. DRAGONETTI, « Le mariage des arts au Moyen Âge », in Littérature et musique, Bruxelles, 1982, p. 59-73, ici p. 59 (Publications des Facultés universitaires Saint Louis, $\left.n^{\circ} 28\right)$. 
2. R. DRAGONETTI, « Le mariage des arts... ", ibid., p. 61.

3. M. Zink, Poèsie et conversion, Paris, 2003.

4. Fixé par MARTIANUS CAPELla dans les Noces de Philologie et de Mercure au V $\mathrm{V}^{\mathrm{e}}$ siècle.

5. Voir les travaux de référence de Paul Zumthor, Michel Zink, Roger Dragonetti.

6. G. DIDI-HUBERMAN, «Imitation, représentation, fonction. Remarques sur un mythe épistémologique ", in L'image. Fonctions et usages des images dans l'Occident médiéval, Paris, 1996, p. 59-86.

7. G. DIDI-HUBERMAN, Fra Angelico. Dissemblance et figuration, Paris, 1995, p. 232.

INDEX

Mots-clés : musicien 